Génét. Sél. Evol., 1986, 18 (1), 11-24

\title{
Variable outcome in competition experiments between Drosophila melanogaster and Drosophila simulans
}

\author{
P. CASARES and María C. CARRACEDO
}

Departamento de Genetica, Facultad de Biologia, Universidad de Oviedo, 33006 Oviedo, Spain

\section{Summary}

Individuals of wild phenotype of Drosophila melanogaster and $D$. simulans, extracted from a single base population of each species, were placed to compete in single monogenerational cultures. Four tests were carried out at different dates, showing that the competitive result was different in each test, with several interspecific interactions that included mutual facilitation as well as mutual inhibition. So, the competitive interactions were not constant throughout the experiment. In the base populations, adult and preadult fitness components underwent profound changes with time, modifying in different ways the relative competitive ability of both species. The competitive outcome measured from laboratory populations was unpredictable.

It is suggested that the observed changes in population fitness and competitive ability in the base populations of the 2 species might be related to the dynamic of seasonal population growth of these species, which is discussed in relation to the distribution and relative abundance of these drosophilids in nature.

Key words: Interspecific competition, Drosophila melanogaster, Drosophila simulans, timedependent fitness, competitive interactions.

\section{Résumé}

Résultat variable dans des expériences de compétition entre Drosophila melanogaster et Drosophila simulans

Des individus de phénotype sauvage de Drosophila melanogaster et $D$. simulans extraits d'une population de base de chaque espèce, ont été utilisés dans des expériences de compétition sur une génération. On a réalisé 4 tests à des dates différentes, obtenant chaque fois un résultat compétitif différent, avec divers types d'interactions interspécifiques qui incluent aussi bien une facilitation réciproque qu'une inhibition réciproque. Ainsi donc, le résultat n'a pas été constant dans le temps. Dans les populations de base, les composantes de la fitness adulte et pré-adulte ont subi d'importants changements dans le temps, modifiant la capacité compétitive relative des 2 espèces. Le résultat de la compétition, évalué à partir des populations de laboratoire, s'est avéré impossible à prévoir.

On suggère que les changements de la fitness et de la capacité compétitive des populations des 2 espèces pourraient être liés à la dynamique de croissance saisonnière, ce qui est discuté par rapport à l'abondance relative et la distribution de ces drosophiles dans la nature.

Mots clés : Compétition interspécifique, Drosophila melanogaster, Drosophila simulans, fitness temps-dépendante, interactions compétitives. 


\section{Introduction}

Interspecific competition is considered by many biologists as an important cause of evolution through natural selection. When 2 newly separated or closely related species compete for scarce ressources there are 2 general trends : one, that the less fit species is eliminated (competitive exclusion) ; the other, that a more or less stable coexistence is established (BARKER, 1983 ; for a recent comment). Competition in both cases causes a selective pressure that may either increase the competitive ability of competitors by different mechanisms or drive both species towards the utilization of alternative resources, the so called, ecological divergence. From an evolutionary point of view, the selection decreasing competition is likely to require a longer time. So, if 2 species actually coexist, it is probable that they will differ in a broad spectrum of ecological determinants.

Drosophila melanogaster and D. simulans are a pair of sibling species that have been useful material for studying competition. They are cosmopolitan, being generally caught in the same locations and with the same baits. Their population sizes suffer seasonal oscillations, with their respective peaks appearing in different months. But in some localities in which $D$. melanogaster was endemic, $D$. simulans appeared as a colonizer displacing in number the otherwise abundant $D$. melanogaster, as has been reported by Hoenigsberg (1968) in Colombia, Tantawy \& Mourad (1970) in Egypt, and Watanabe \& KaWANishi (1976) in Japan. These reports are very different to the results found in the laboratory, where $D$. melanogaster appears to be superior to $D$. simulans in most of the components of darwinian fitness considered as important. Notably, this also occurs when the above mentioned populations from Egypt are examined in the laboratory (TANTAWY \& MouraD, 1970). Taking these facts into account, it is clear that we do not know the really important factors in determining the fitness of a population.

But, do these 2 species really compete? If so, with what intensity ? No direct evidence from nature is known, but competition may be inferred (BARKER, 1983) because, when sympatric, some fruits are used in association. However, the colonization of Japan by $D$. simulans and the parallel decrease in number of $D$. melanogaster can occur although competition between them appears to be scanty. Certainly, if niche overlap between the 2 species is small and if they compete for limited resources, then coexistence would be possible even though one species might reduce the population size of the other.

Some ecological differences have been found under laboratory conditions between larvae (Barker, 1971), pupae (Sameoto \& Miller, 1968 ; BArker, 1971 ; Manning \& Markow, 1981 ; Casares \& Rubio, 1984 ; Casares \& Carracedo, 1984 a ; 1984 b) and adults (McDonald \& Parsons, 1973 ; Ali \& El-Helw, 1974 ; Parsons, 1975 a ; Kawanishi \& Watanabe, 1978 ; Kawanishi \& LeE, 1978). Therefore, we cannot rule out the possibility that competition between these 2 species in nature may be less intense than is commonly accepted, due to the fact that a great ecological divergence may exist between them.

In this paper, we present results coming from a competition study between $D$. melanogaster and $D$. simulans. We have considered the use of freshly caught populations and flies of wild phenotype to be essential. Several components of fitness have been recorded in order to obtain a general view of the interspecific interactions, and an evaluation of the relative importance of both adult and preadult stages. 


\section{Material and methods}

The biological material consisted of a population of $D$. melanogaster and another of D. simulans freshly caught in 2 neighbouring localities of Asturias (Spain). Each population was kept in two 3 litre population cages, which allows more than 800 flies per population. The populations were kept under laboratory conditions with illumination and temperature that were partially parallel to diurnal and seasonal oscillations. The renovation of the cage's food vials was done when the experimenter judged that a generation had emerged form the vials, that is, at time intervals fixed by the dynamics of each species. No mutants were employed ; all the experiments were performed with wild flies obtained from the population cages.

Control and competition cultures were simultaneously initiated, the controls with adult densities of 8 and 16 pairs of flies, named M8 and M16 for D. melanogaster and S8 and S16 for D. simulans. The mixed competition cultures, C16, were made with 8 pairs of each species and, therefore, with a 1:1 ratio. The experimental design is summarized as follows : adult virgin flies developed in bottles under constant density, and aged up to 5 days, were introduced into vials $(25 \times 120 \mathrm{~mm})$, without anaesthesia, in the required numbers and species proportions. Then, the number of matings occurring in a period of 2 hours was recorded. Later, the adults were put into vials with food, and allowed to lay eggs during 3 consecutive 24-h periods, and were changed to a fresh vial at the end of each period. Food was extracted from vials. The laid eggs were counted using a stereoscopic microscope, and food returned to vials to allow egg to adult development. Data from the eggs and adults scored in the first 48 hours (two vials), were used as the fecundity and productivity values. Data from the third 24 hours period (one vial) were used to estimate the egg-adult viability for both control and competition cultures. All the tests were replicated with a minimum-maximum number of 6-9 for controls and 24-37 for mixed cultures. These values were obtained throughout 4 experimental blocks, named I, II, III and IV, carried out consecutively in April 1977, August 1977, November 1977 and March 1978.

The food used had the following ingredients : Baker's yeasts $(10 \mathrm{p} .100)$, sucrose (10 p. 100), agar (1.2 p. 100), salt (0.05 p. 100) and propionic acid (0.05 p. 100). All the experiments were carried out under constant light, at $21.5 \pm 0.5^{\circ} \mathrm{C}$.

\section{Results}

Table 1 shows the mean values of productivity of the control cultures. Two facts are remarkable : firstly, the great differences in productivity between the experimental blocks, with both species showing the highest productivity in block IV. Secondly, the productivity of controls M16 and S16 is far from reaching twice the productivity found in the M8 and S8 controls. Thus, productivity is density dependent. The 2 species suffer a strong intraspecific competition in the density we employed. 
TABLE 1

Top, mean productivity (number of replications in brackets) of D. melanogaster and D. simulans in control cultures at 2 densities. Bottom, productivity of competition cultures showing the mean values of D. melanogaster, D. simulans and total productivity separately.

Right, the error variances with 64 degrees of freedom each. Comparisons were made by a Student's " $t$ " test.

\begin{tabular}{|c|c|c|c|c|c|}
\hline & \multicolumn{4}{|c|}{ Blocks } & \multirow{2}{*}{ Variance $\#$} \\
\hline & I & II & III & IV & \\
\hline \multicolumn{6}{|l|}{ Controls of D. melanogaster } \\
\hline$\ldots \ldots \ldots$ & $92.3(6)$ & $100.8(6)$ & $77.8(10)$ & $120.8(11)$ & 165.6 \\
\hline$\ldots \ldots$ & $101.4(8)$ & $114.8(7)$ & $121.3(9)$ & $193.1(8)$ & \\
\hline \multicolumn{6}{|l|}{ Controls of $D$. simulans } \\
\hline$\ldots$ & $35.5(5)$ & $56.0(4)$ & $34.8(8)$ & $55.8(8)$ & 94.5 \\
\hline S16 & $69.3(7)$ & $64.0(4)$ & $60.1(8)$ & $109.0(8)$ & \\
\hline \multicolumn{6}{|l|}{ Competition : } \\
\hline $\begin{array}{l}\text { D. melanogaster } \ldots \ldots \\
\text { Comparison vs } \cdot 1 / 2 \cdot \mathrm{M} 16\end{array}$ & $\begin{array}{l}64.8(24) \\
\mathrm{t}=2.9^{* *}\end{array}$ & $\begin{array}{l}71.5(16) \\
t=2.5^{*}\end{array}$ & $\begin{array}{l}58.0(35) \\
\mathrm{t}=0.5\end{array}$ & $\begin{array}{l}87.9(37) \\
t=1.6\end{array}$ & 165.6 \\
\hline $\begin{array}{l}\text { D. simulans } \cdots \cdots \\
\text { Comparison vs } \cdot 1 / 2 \cdot S 16 \ldots \ldots\end{array}$ & $\begin{array}{l}25.4(24) \\
t=2.1^{*}\end{array}$ & $\begin{array}{l}45.2(16) \\
t=2.4^{*}\end{array}$ & $\begin{array}{c}29.4(35) \\
t=0.1\end{array}$ & $\begin{array}{l}50.2(37) \\
\mathrm{t}=1.1\end{array}$ & 94.5 \\
\hline $\begin{array}{l}\text { Total ......... } 1 / 2(\mathrm{M} 16+\mathrm{S} 16) \\
\text { Comparison vs } \ldots\end{array}$ & $\begin{array}{l}90.2(24) \\
\mathrm{t}=0.9\end{array}$ & $\begin{array}{l}116.7(16) \\
t=2.8^{* *}\end{array}$ & $\begin{array}{c}87.4(35) \\
t=0.5\end{array}$ & $\begin{array}{l}138.1(37) \\
t=1.8 £\end{array}$ & 305.8 \\
\hline
\end{tabular}

(\#) With 64 degrees of freedom. $\left({ }^{*}\right) p<0.05 .\left(^{* *}\right) p<0.01$. (f) $p=0.07$.

In addition, table 1 shows the productivities of $D$. melanogaster and $D$. simulans in the C16 competition cultures, separately. A useful method to ascertain the possible involvement of competitive interactions, is to compare the value observed in competition with an expected value obtained from the controls at the same adult density (Futuyma, 1970 ; Barker, 1971 ; Wallace, 1974), in this case, M16 and S16. In this way, some comparisons were made separately in each block, assuming the same variance of error for the expected value as for the value observed in competition cultures. From table 1 we can infer that the productivity of $D$. melanogaster in competition in block $I$ is significantly higher than the productivity of the control, i.e., intraspecific is stronger than interspecific competition, which denotes the existence of a remarkable interspecific facilitation of this species when competing with $D$. simulans. In clear contrast, the productivity of $D$. simulans in competition is lower than expected, since its productivity is inhibited by $D$. melanogaster. In this species, interspecific proves to be stronger than intraspecific competition. Thus, an interspecific facilitationinhibition is detected in block $\mathrm{I}$, with $D$. melanogaster obtaining a gain at the expense of $D$. simulans when these species compete for limited resources.

A different result appears in block II : in competition, D. melanogaster as well as $D$. simulans increased their productivities with respect to controls, which we can refer to as mutual interspecific facilitation. It is noteworthy that the productivity of $D$. simulans in competition in block $I$ is 85 p. 100 lower than the control, but 40 p. 100 
higher than the respective control in block II. Consequently, the competitive ability of D. simulans was very different in each block.

The preceeding results contrast with block III, where no species modified its productivity when developed in the same culture and this indicates non-interference between them, i.e., the limited resources were equally shared by the competitors.

Finally, the observed-expected differences found for each species in block IV are not significantly different at the $5 \mathrm{p}$. 100 level, but when the total productivity is compared, the difference shows 7 p. 100 probability ; this suggests that, in block IV, the 2 species undergo a slight mutual inhibition when they are in competition.

The most important conclusion is the existence of different competitive results from one block to another. In the 4 blocks, temperature, food and methodology were exactly the same, the only difference being the time at which they were achieved. In each block, the adults came from the same population cages kept under laboratory conditions. What is the explanation for the different competitive outcomes ? In each of the 4 blocks, the number of pairings recorded during the first 2 hours of courtship, the number of eggs laid in 48 hours and the egg-adult viability were estimated. Now, these can be examined to determine their relative importance in giving rise to the above mentioned variable competitive results.

The number of pairings recorded in 2 hours may be considered as an estimation of mating speed, and if this important component of fitness (EHRMAN \& Parsons, 1976) were modified by interspecific interaction during courtship, the productivity in competition could be lower than in controls. Table 2 shows the percentages of pairing observed in control and competition cultures. The comparisons between densities (tabl. 2, sections $\mathrm{A}$ and $\mathrm{B}$ ) showed that, in D. simulans, the percentage of mating was not modified by increasing adult density. Similar results were observed for $D$. melanogaster in blocks II and IV, whereas in blocks I and III, the percentages of mating decreased when adult density increased, which denoted the existence of intraspecific mating interference in this species. Because of this result, the percentages of mating in competition, C16 (tabl. 2, section C), were contrasted with expected values obtained using the M16 and S16 controls, carried out, therefore, at the same 16-density. The single expected value for $D$. simulans was calculated as the weighed mean of the 4 nondifferent blocks (tabl. 2, section B). For D. melanogaster, 2 different expected values were employed : one, by weighting the means of the non-different I, II and IV blocks ; the other corresponding to the statistically different mean of block III. Table 2, (section C) reveals that none of the observed-expected differences were significant. In conclusion, the different "between-blocks " competitive responses in productivity shown in table 1 , can not be explained by differences in the number of matings found in control versus competitive cultures.

However, interspecific mating interference was apparent in a simultaneous experiment made with the same populations and identical culture conditions : Table 2 (section D) shows the percentages of mating achieved by 8 virgin pairs from one species in the presence of 8 newly mated pairs of the other species, during the first 2 hours of courtship. These percentages were contrasted with the respective controls and significant differences were only observed in block III. Thus, in this block the presence of one of the 2 mated species causes an interspecific interference in courtship in the other, a feature that does not occur in the other 3 blocks. This is another result showing the large differences in components of fitness exhibited by the flies in the 4 blocks of the present work. 


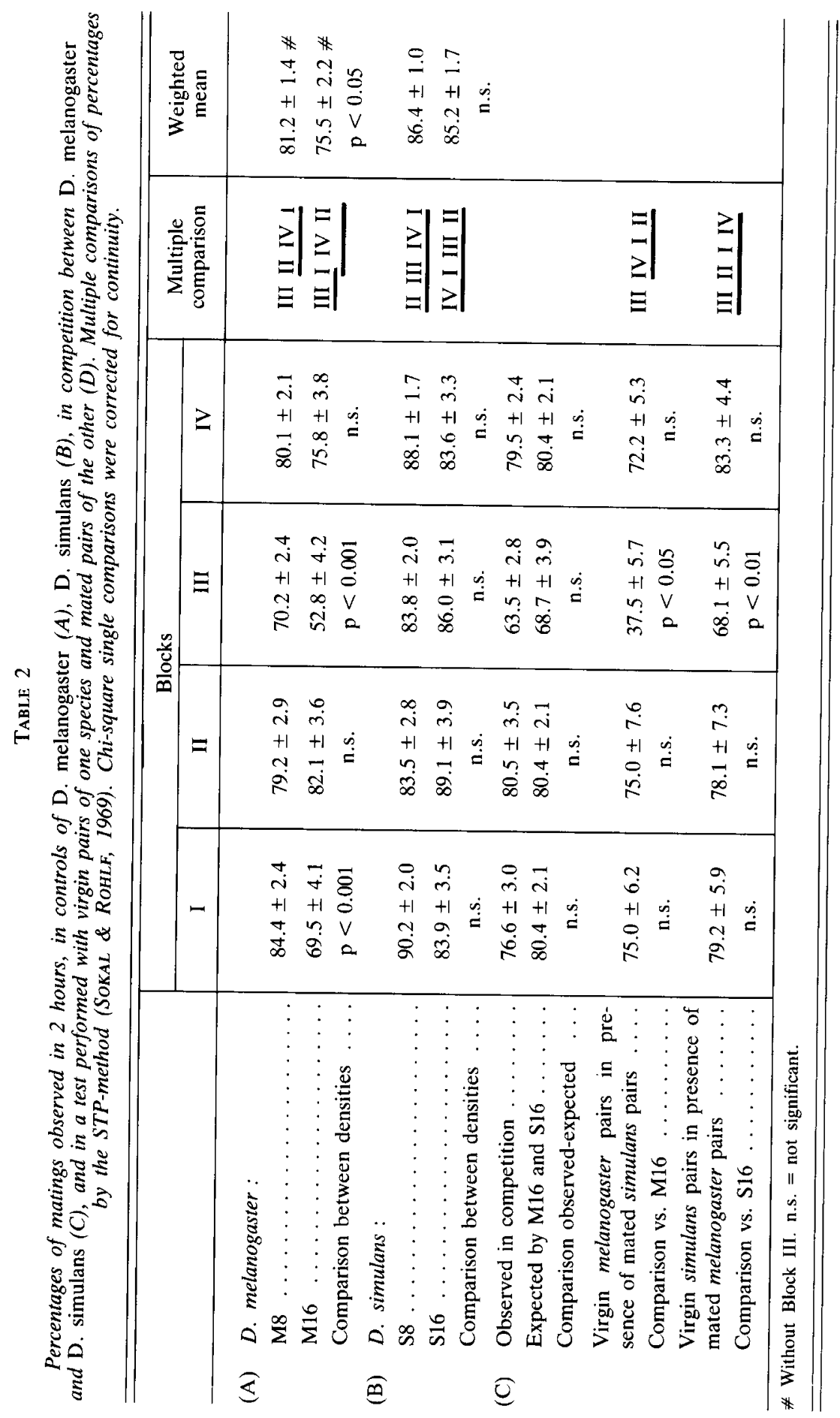


The fecundity values of controls and competition cultures (no data were obtained in block I) are given in table 3. The 3 comparisons between the expected average value from controls M16 and S16, and the observed value in competition were not significant. However, the values of fecundity show parallelism with the values of productivity (tabl. 1) which suggests that the mutual facilitation in block II or the mutual inhibition in block IV, could be caused by different interspecific interactions during the oviposition process.

It is interesting to emphasize another difference between the 2 species : since the control adult density was increased 100 p. 100 from M8 and S8 to M16 and S16, the fecundity should increase by the same percentage, unless some limiting factor is operating. Nevertheless, as appears in table 3 in parenthesis, D. melanogaster increased its fecundity nearly 50 p. 100 in each block whereas in $D$. simulans, the fecundity rose nearly the expected 100 p. 100 in blocks III and IV, in contrast with block II where 16 females laid only 11 p. 100 more eggs than 8 females. This behavior appears to be normal when the oviposition sites are scarce (AshBuRner, 1978, for references). But it is interesting that in our paper the strongest inhibition in the oviposition of $D$. simulans occurred in block II although the highest value of fecundity and the greatest food saturation by eggs, was found in block IV.

TABLE 3

Mean fecundity of $\mathrm{D}$. melanogaster and $\mathrm{D}$. simulans in controls and mean fecundity observed and expected for both species in competition. In addition, the percentage increase in fecundity for each block and species when density increased from 8 to 16 females (100 p. 100 being expected). Numbers of replications are given in brackets.

\begin{tabular}{|c|c|c|c|}
\hline & \multicolumn{3}{|c|}{ Blocks } \\
\hline & II & III & IV \\
\hline \multicolumn{4}{|l|}{ D. melanogaster : } \\
\hline$\ldots \ldots \ldots \ldots \ldots \ldots \ldots \ldots \ldots \ldots \ldots$ & $135.3(3)$ & $92.3(6)$ & $178.0(4)$ \\
\hline 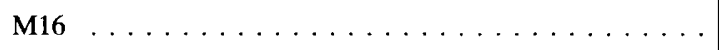 & $186.0(4)$ & $156.0(5)$ & $283.0(4)$ \\
\hline Increase $\ldots \ldots \ldots \ldots \ldots \ldots \ldots \ldots$ & $37 \%$ & $69 \%$ & $59 \%$ \\
\hline \multicolumn{4}{|l|}{ D. simulans : } \\
\hline S8 $\ldots \ldots \ldots$ & $75.5(4)$ & $55.2(6)$ & $103.5(4)$ \\
\hline $\mathrm{s} 16 \ldots \ldots \ldots \ldots \ldots \ldots \ldots$ & $87.5(4)$ & $107.7(4)$ & $210.7(4)$ \\
\hline Increase $\ldots \ldots \ldots \ldots \ldots \ldots \ldots$ & $11 \%$ & $95 \%$ & $104 \%$ \\
\hline \multicolumn{4}{|l|}{ Competition : } \\
\hline C16 observed & $166.1(8)$ & $136.0(19)$ & $226.5(20)$ \\
\hline Expected by $1 / 2(\mathrm{M} 16+\mathrm{S} 16)$ & 136.7 & 131.9 & 246.9 \\
\hline \multicolumn{4}{|c|}{ Error variance : 687.6 (35 degrees of freedom) } \\
\hline
\end{tabular}

The last group of data recorded was the egg-adult viability obtained from control and competition cultures. For each species and block, a linear regression of adults on eggs was estimated, and results appear in table 4 . The mean values of laying were different between blocks and between species within blocks. For this reason, for 
comparing the preadult viabilities between control and competition cultures, we calculated a fixed value of laying for each block, as the average of the mean values of laying of each species in controls ( $\bar{X}$ in tabl. 4). These fixed values were 43,50 and 80 eggs for blocks II, III and IV respectively, and they were put in the regression equations to obtain the preadult-viability averages for competition and control cultures. These are shown in table 4 as percentages. Clearly, the preadult viability in competition is not different to the average viability of $D$. melanogaster - $D$. simulans. That is, there is no interspecific interaction at the preadult level, intraspecific being as intense as interspecific competition. Hence, we can conclude that the appearance of variable competitive results are not due to the occurrence of different intensities or different kinds of interference during the preadult competition in the 4 experimental blocks. Furthermore, a new and surprising result is observed : in controls, the regression lines of blocks II and III of $D$. melanogaster, and II of $D$. simulans, pass over the origin 0,0 (intercept, " a ", non significant) revealing that in these blocks the egg-adult viability is constant along the range of egg density observed, viability being density independent. This is not the case with blocks IV of D. melanogaster and III and IV of D. simulans (a,

TABLE 4

Regression of adults $(Y)$ on eggs $(X)$ in each experimental block for control and competition cultures. The regression coefficients (b) and the intercepts (a) are given with their standard errors. From the equations, an egg-adult viability was calculated for a fixed $(X)$ number of eggs. These numbers were obtained in each block as the average of mean fecundity $(\bar{X})$ of each species in controls.

\begin{tabular}{|c|c|c|c|}
\hline & \multicolumn{3}{|c|}{ Blocks } \\
\hline & II & III & IV \\
\hline \multicolumn{4}{|l|}{ Controls of $D$. melanogaster: } \\
\hline Mean point, $\bar{X}-\bar{Y} \ldots \ldots$ & $46.7-36.4$ & $54.2-49.3$ & $82.4-61.0$ \\
\hline Regression $b \pm$ s.e. $\ldots \ldots \ldots \ldots \ldots$ & $0.75 \pm 0.06^{* * *}$ & $0.86 \pm 0.03^{* * *}$ & $0.68 \pm 0.02^{* * *}$ \\
\hline$a \pm$ s.e. $\ldots \ldots \ldots \ldots \ldots$ & $1.20 \pm 3.80$ & $2.86 \pm 1.91$ & $5.26 \pm 1.78^{*}$ \\
\hline Egg-adult viability $\ldots \ldots \ldots \ldots \ldots \ldots$ & $78 \%$ & $92 \%$ & $74 \%$ \\
\hline For $\mathrm{X}$ equal to $\ldots \ldots \ldots \ldots \ldots$ & 43 & 50 & 80 \\
\hline \multicolumn{4}{|l|}{ Controls of $D$. simulans } \\
\hline Mean point, $\overline{\mathrm{X}}-\overline{\mathrm{Y}}$ & $38.8-31.3$ & $45.6-25.3$ & $77.3-44.0$ \\
\hline Regression $b \pm$ s.e. & $0.76 \pm 0.12^{* * *}$ & $0.40 \pm 0.04^{* * *}$ & $0.44 \pm 0.05^{* * *}$ \\
\hline$a \pm$ s.e. $\ldots \ldots \ldots \ldots$ & $1.74 \pm 4.96$ & $7.30 \pm 1.20^{* * *}$ & $10.10 \pm 4.24^{*}$ \\
\hline Egg-adult viability $\ldots \ldots$ & $80 \%$ & $54 \%$ & $56 \%$ \\
\hline For $\mathrm{X}$ equal to $\ldots \ldots \ldots \ldots \ldots$ & 43 & 50 & 80 \\
\hline \multicolumn{4}{|l|}{ Competition : } \\
\hline Mean point, $\bar{X}-\bar{Y}$ & $59.1-41.8$ & $70.6-49.7$ & $102.7-60.5$ \\
\hline Regression $b \pm$ s.e. . . & $0.42 \pm 0.04^{* * *}$ & $0.52 \pm 0.05^{* * *}$ & $0.43 \pm 0.06^{* * *}$ \\
\hline$a \pm$ s.e. . & $16.72 \pm 2.51^{* * *}$ & $13.23 \pm 3.43^{* * *}$ & $16.69 \pm 6.75^{*}$ \\
\hline Egg-adult viability & $81 \%$ & $78 \%$ & $63 \%$ \\
\hline For $\mathrm{X}$ equal to & 43 & 50 & 80 \\
\hline
\end{tabular}

$\left(^{*}\right) \mathrm{p}<0.05 .\left(^{* * *}\right) \mathrm{p}<0.001$. 
significant), whose egg-adult viabilities decrease when egg-density increases. This event suggests that larvae of different blocks possess very distinct efficiencies of getting the same nutrients, since in the first group of blocks, the egg-adult viability is constant, that is, density independent, whereas in the second group of blocks the viability is inversely dependent on egg density. Notably, this block-dependent effect is not parallel in the 2 species. More notable is the fact that, in $D$. simulans, although blocks II and III show a similar egg density (tabl. 3), in the former, the egg-adult viability is low and decreases with density, whereas the latter shows a high and constant egg to adult viability. So, the larval fitness was very different in each block.

\section{Discussion}

The results of table 1 show that in the 4 blocks conducted at different times, different competitive responses exist between $D$. melanogaster and $D$. simulans. To explain this, we have looked for a relation between these results and some fitness components obtained in the same blocks. However, neither the number of matings recorded in 2 hours nor the preadult viability can explain the variable competitive outcomes. Female fertility, which was recorded after the period of laying, showed no differences between both densities and species, or between control and competitive cultures (CASARES, 1983) and so, female fertility was not able to explain the results of table 1 either.

The possibility that distinct larval interspecific interactions may be the origin of the observed competitive results is also discarded as much by the results of an experiment made with these same populations some months after ending block IV (CASARES \& Rubio, 1984), as by the results of Miller (1964) and BARKER (1967, 1971), all of which point towards an ecological equivalence, especially at intermediate density, when larvae of $D$. melanogaster and $D$. simulans are developed together.

Fecundity seems to be the only parameter related to the interspecific mutualfacilitation, non-interference and mutual-inhibition in productivity, found in our paper. Therefore, the most acceptable hypothesis is that the behaviour of both species during the oviposition process has played a preponderant role in determining the variable competitive results reported here. This suggests that, in the course of time, different interspecific interactions occurred during oviposition. This supposition seems to be confirmed by the results obtained in February and April of 1979 using the same base populations as those described here, when it was shown that virgin females of any of the 2 species partially inhibited the oviposition of fertile females of the other species (CASARES, 1984), but with an intensity and an interspecific interaction that were different according to the month in which the tests were done. But the question is why, in our paper, interspecific interaction in oviposition varies with time.

If we review the literature on competition between the 2 siblings, different competitive results appear : Furuyma (1970) found facilitation for D. melanogaster and inhibition for $D$. simulans; BARKer \& PODGER (1970) reported inhibition of $D$. melanogaster and facilitation of $D$. simulans, in contrast with FUTUYMA. Later, BARKER (1971) working with the same strains and experimental conditions, observed mutual facilitation ; HEDRICK (1973) found that one strain of $D$. melanogaster was inhibited and another facilitated when faced with the same $D$. simulans strain. In clear contrast, 
Wallace (1974) described non-interference between the 2 species. In short, several competitive results are known when $D$. simulans and $D$. melanogaster compete in the laboratory, which may be attributed to the genetic diversity of the strains employed by the authors mentioned. But it is important that none of these authors replicated their experiments at different times.

The influence of the experimental design upon the results obtained can not be rejected : BARKER (1971) mainly ascribed his competitive result to a pupal interaction, whereas HEDRICK (1973) noted that his results were largely due to the duration of development. But in our paper, the methodology was exactly the same in the 4 blocks and so the same relative specific fitness between the 2 species should be expected. This is not the case. Some examples : the highest preadult viability of $D$. melanogaster appeared in block III whereas this occurred for $D$. simulans in block II. In $D$. simulans, block IV, with the largest egg density, did not show the smallest preadult viability as might be expected on account of the more intense intraspecific competition. For both species, the major homo- and hetero-specific interaction in courtship (measured by mating speed) appeared in block III, and despite this, it was the only one in which no interspecific interaction in productivity was detected (tabl. 1). In regard to fecundity, a remarkable inhibitory behaviour in the oviposition of $D$. simulans, probably due to food saturation, was noted in block II, in which 16 females laid almost the same number of eggs as 8 females; but this inhibitory behaviour did not occur in block IV, although fecundity (and food saturation) was much higher in the latter. No similar facts were found in $D$. melanogaster. Other between-blocks interspecific differences have been presented in Results. To summarize, neither the competitive fitness of the 2 species nor the competitive outcome were constant through time. A clear species-block interaction is apparent.

Our results are troublesome. What is the meaning of these repeated variations in the estimates of several independent components of fitness? Why is competitive outcome block-dependent? Three possible explanations are. One, that uncontrolled environmental variations had been operating causing in each block the appearance of different values of mating, fecundity, productivity and competitive ability, and notably, with a very distinct effect in $D$. melanogaster and $D$. simulans. If correct, the competitive outcome between these species, when measured from monogenerational tests at a given time, would be simply unpredictable. Two, that the different competitive outcomes could be imputed to species-specific cyclic (seasonal ?) endogenous changes in the physiology of the adult flies; to prove this, we would need to study additional seasonal cycles of competition. Three, that the base populations had suffered changes in their genetic composition at random or by means of selective processes. Any one of these possibilities, or the 3 , may be true.

It is well known that in nature, $D$. simulans and $D$. melanogaster have their respective population peaks at different seasons (PARsons, $1975 \mathrm{~b}$, for a review) with $D$. melanogaster being more abundant in early summer and $D$. simulans in late summer and autumn. McKenzIE \& PARSons (1974) have observed that the population size ratio of melanogaster/simulans oscillates depending on the monthly mean temperature. Summer temperature regulated the population size of each species in Japan (WATANABE et al., 1984). As far as we know, no laboratory study has been made with artificial seasonal climatic oscillations. Our base populations were kept in the laboratory, and submitted to natural daily and seasonal variations of temperature. These variations have generated in the base populations of each species, and over the year, shorter generation times and larger population sizes in spring and summer than in winter and autumn. 
This suggests the existence of different dynamics of populational growth in each block. So, we are tempted to speculate that the observed changes in the relative competitive fitness of the 2 species could be related to the natural spring-summer-autumn cycles of population growth. This cannot be properly tested with the results shown here, but the hypothesis is attractive and worthy of broader experimental work.

In studies on the evolution of competitive ability in mixtures of closely related species in which 2 or more species compete over a long period of time (see BARKER, 1983, for a review), the performance of selection lines, i.e., mixed cultures, is compared with that of control lines after several generations of competition, with some results that claimed the existence of changes in competitive ability developed by natural selection. Our results have shown that individuals of $D$. melanogaster and $D$. simulans extracted from the base populations at different times, show very different competitive abilities, with some fitness components showing profound changes with time. So, as pointed out by BARKER (1983), it is difficult to prove whether the above mentioned changes in competitive ability in lines presumably selected for it, have been directly originated by the competitive process, since the control lines can also suffer changes that, as in our results, may alter the competitive result (BARKER, 1973 ; HEDRICK, 1973).

\section{Conclusion}

1) The competitive outcome is always favorable to D. melanogaster due to a higher reproductive fitness than its sibling $D$. simulans.

2) Different kinds of interspecific interaction appear at different times. Therefore, the competitive relative fitness is not constant in our populations of $D$. melanogaster and $D$. simulans.

3) An interaction between species, blocks and fitness components is apparent. The estimates of mating, fecundity, and egg-adult viability, the oviposition behaviour, larval fitness, and their responses to increased density, varied in an unpredictable way according to the block they were measured in.

4) A relation between the performance of a species in monocultures and its competitive ability was not found.

Received January 24, 1985.

Accepted September 18, 1985.

\section{Acknowledgements}

We wish to thank Drs. Francisco J. Alaya, J.S.F. Barker and Andrés Moya for their critical reading of the manuscript, which greatly benefits from their valuable comments. 


\section{References}

All A.M.M., El-Helw M.R., 1974. Differences in the yeasts preferred by D. melanogaster and D. simulans. Egypt. J. Genet. Cytol, 3, 204-210.

Ashburner M., Thompson J.N. Jr., 1978. The laboratory culture of Drosophila. In : Ashburner M., Wright T.R.F. (ed.), "The Genetics and Biology of Drosophila ", Vol. 2 a, 2-109. Academic Press, London.

BARKer J.S.F., 1967. The estimation of relative fitness in Drosophila populations. V. Generation interval and heterogeneity in competition. Evolution, 21, 299-309.

BARKER J.S.F., 1971. Ecological differences and competitive interactions between $D$. melanogaster and D. simulans in small laboratory populations. EEcologia, 8, 139-156.

BARKER J.S.F., 1973. Natural selection for coexistence or competitive ability in laboratory populations of Drosophila. Egypt. J. Genet. Cytol, 2, 288-315.

Barker J.S.F., 1983. Interspecific competition. In : Ashrurner M., Wright T.R.F. (ed.). "The Genetics and Biology of Drosophila», Vol. 3 c, 285-341. Academic Press, London.

Barker J.S.F., Podger R.N., 1970. Interspecific competition between D. melanogaster and $D$. simulans. Ecology, 51, 855-864.

Casares P., 1983. Influencia de la densidad sobre la biologia reproductora de las especies gemelas D. melanogaster y D. simulans (Insecta : Diptera.) Boletin Ciencias Naturales I.D.E.A., 31, 191-210.

CASARes P., 1984. Interspecific inhibition between $D$. melanogaster and $D$. simulans during oviposition process. Drosophila Inf. Serv., 60, 69-71.

Casares P., Carracedo M.C., 1984 a. Comportamiento de pupacion en $D$. melanogaster y $D$. simulans. I. Respuestas ante el cambio de humedad y el tipo de alimento. Revista Biologia Univ. Oviedo, 2, 11-20.

Casares P., Carracedo M.C., 1984 b. Comportamiento de pupacion en $D$. melanogaster and $D$. simulans. II. Influencia de la humedad en la altura de pupación. Revista Biologia Univ. Oviedo, 2, 21-28.

Casares P., Rubio J., 1984. Competencia interespecífica entre los preadultos de D. melanogaster y D. simulans. Medio Ambiente, 7, 3-8.

Ehrman L., Parsons P.A., 1976. The Genetics of Behavior. 390 pp. Sinauer Ass., Massachusetts.

Futuyma D., 1970. Variation in genetic responses to interspecific competition in laboratory populations of Drosophila. Amer. Nat., 104, 239-252.

HedRICK P., 1973. Factors responsible for a change in interspecific competitive ability in Drosophila. Evolution, 26, 513-522.

HoENIGSBERg H.F., 1968. An ecological situation which produced a change in the proportion of $D$. melanogaster to D. simulans. Amer. Nat., 102, 389-390.

Kawanish M., LeE W., 1978. Food preferences of D. simulans and D. melanogaster. Jap. J. Ecol., 28, 231-235.

Kawanishi M., Watanabe T.K., 1978. Differences in photo-preferences as a cause of coexistence of D. simulans and D. melanogaster in nature. Jap. J. Genet., 53, 209-214.

Manning A., Markow T.A., 1981. Light-dependent pupation site preference in Drosophila. II. D. melanogaster and D. simulans. Behav. Genet., 11, 557-563.

McDonald J., Parsons P.A., 1973. Dispersal activities of the sibling species D. melanogaster and D. simulans. Behav. Genet., 3, 293-301.

McKenzie J.A., Parsons P.A., 1974. Numerical changes and environmental utilization in natural populations of Drosophila. Aus. J. Zool., 22, 175-187.

Miller R.S., 1964. Larval competition in D. melanogaster and D. simulans. Ecology, 45, 132-148.

Parsons P.A., 1975 a. Phototactic responses along a gradient of light intensities for the sibling species D. melanogaster and D. simulans. Behav. Gen., 5, 17-25.

Parsons P.A., 1975 b. The comparative evolutionary biology of the sibling species $D$. melanogaster and D. simulans. Q. Rev. Biol., 50, 151-169. 
SAmeoto D.D., Miller R.S., 1968. Selection of pupation site by $D$. melanogaster and D. simulans. Ecology, 49, 177-180.

Soxal R.R., Rohlf F.J., 1969. Biometry. The principles and Practice of Statistics in Biological Research. 778 pp., Freeman, San Francisco.

Tantawy A.O., Mourad A.M., 1970. Studies on natural populations. VIII. A note on the directional changes over a long period of time in the structure of Drosophila near Alexandria, Egypt. Amer. Nat., 104, 105-109.

Wallace B., 1974. Studies on intra- and inter-specific competition in Drosophila. Ecology, 55, 227-244.

Watanabe T.K., Kawanishi M., 1976. Colonization of D. simulans in Japan. Proc. Jap. Acad., 52, 191-194.

Watanabe T.K., Inoue Y., Watada M., 1984. Adaptation of Drosophila simulans in Japan. Jap. J. Genet., 59, 225-235. 